\title{
Road-Feature-Based Multiparameter Road Complexity Calculation Model of Off-Road Environment
}

\author{
Yanan Zhao $\mathbb{D}$, Zhiwei Li $\mathbb{D}$, Li Gao $\mathbb{D}$, and Jian Xiong $\mathbb{C}$ \\ School of Mechanical Engineering, Beijing Institute of Technology, Beijing 100081, China \\ Correspondence should be addressed to Yanan Zhao; zyn@bit.edu.cn
}

Received 3 May 2018; Revised 26 August 2018; Accepted 10 October 2018; Published 25 October 2018

Academic Editor: Mahmoud Mesbah

Copyright (c) 2018 Yanan Zhao et al. This is an open access article distributed under the Creative Commons Attribution License, which permits unrestricted use, distribution, and reproduction in any medium, provided the original work is properly cited.

\begin{abstract}
To promote the development of unmanned ground vehicle technologies, it is necessary to design a scientific and reasonable test method. Road is an important part of test environmental elements, and different road conditions can examine the adaptability of unmanned ground vehicles to the environment. Therefore, the scientific calculation of road complexity is of great importance. Previous studies on road are mainly based on the concept of road roughness; however due to the unicity of road feature indicators, road complexity can only be reflected to a certain extent. This paper proposes a new road-feature-based multiparameter road complexity calculation model of off-road environment to show the complexity of road more comprehensively. First, a multi-sensorbased data acquisition mobile platform is established to obtain more complete road data. Then, based on the analysis of road feature, road indicators like three-dimensional scale, average slope, and adhesion characteristics of travelable area are obtained. According to the analysis methods of road roughness, the principle of analytic hierarchy process, and the data collected from off-road environment, the calculation model of road complexity is determined. Finally, by calculating complexities of several cross-country roads, the feasibility of this model is verified, which provides a theoretical support for the scientific calculation and quantitative analysis of different road complexities.
\end{abstract}

\section{Introduction}

With the rapid development of unmanned ground vehicle (UGV), more and more competitions and tests have been carried out at home and abroad to evaluate the level of UGV $[1,2]$, but no unified evaluation system has been formed yet. The level of UGV is usually determined by three aspects [3]: environment complexity (EC), mission complexity (MC), and human intervention (HI), wherein EC is the most basic aspect.

As an important part of test environmental elements [4], road has a major impact on test and evaluation for UGV [5]. By setting different road conditions, the ability of UGV to adapt to different environments can be examined. For example, in DARPA Grand Challenge 2004, road conditions included well-paved roads, zigzags, hills, swamps, sharp turns, and steep slopes [6], where the performance for UGV to drive autonomously and avoid obstacles in off-road environment was examined; but no vehicle completed the game. In DARPA Grand Challenge 2005 and also in off-road environment, five teams completed the whole process [7] and the ability for vehicles to adapt to off-road environment improved significantly. DARPA Urban Challenge was held at a now-closed air force base to simulate urban traffic environment, focusing on the autonomous driving capacity of UGV in structured environment [8]. "Future Challenge" has been hosted since 2009 [9], where the venues were designed in urban road environment and rural road environment to assess the $4 \mathrm{~S}$ performance (i.e., safety, smartness, smoothness, and speed) of UGV [10], respectively. The Unmanned Ground System (UGS) Challenge conducted by Chinese Army has been carried out in off-road environment since 2014 [11], where the testing environments included muddy uneven roads and part of hardened roads, so that the comprehensive adaptability of UGV in off-road environment can be assessed. It is due to changes in the road environment, in other words, changes in the complexity of road, that higher performance requirements have been put forward for UGV. UGV needs to improve its performance to comply with traffic rules on structured roads [12] and to ride smoothly on unstructured roads. 
In order to analyze the road elements quantitatively, "road complexity" is introduced to characterize the complexity of road environment. So far, few studies on road complexity have been carried out at home and abroad, and the research on road complexity in off-road environment is a huge gap. The research on roads till now is mainly based on the concept of road roughness: ASTM E867 standard defines roughness as "the deviation of a surface from a true planar surface with characteristic dimensions affecting vehicle dynamics and ride qualities" [13]. Researchers and organizations usually use international roughness index (IRI), flatness standard deviation, power spectral density (PSD) of profile elevations, and other statistical indicators to analyze road roughness [14]. IRI defined as the integral of the absolute rare difference between the simulated sprung and unsprung mass motion per unit of distance travelled [15] is essentially a computer-based virtual response-type system based on the response of a quartercar vehicle model as it traverses a tested pavement section at a constant speed of $80 \mathrm{~km} / \mathrm{h}$ [16]. PSD, a mathematical representation of the spatial wavelength composition of road profiles rather than a summary index of roughness [17], is calculated by the equation provided by ISO 8608 standard [18]; according to PSD, road roughness is divided into eight different classes. Dynamic load index (DLI), representing truck dynamic loads transmitted to road pavements due to the presence of irregularities on pavements surface [19], is calculated as a weighted index of variances of the profile elevations in the frequency ranges of 1.5-4 and 8-15 Hz, where the first frequency range corresponds to truck body bounce, the second frequency range to axle bounce [20]. However, whether it is IRI, PSD, or DLI, it can only reflect the road complexity to a certain extent. Therefore, it is necessary to propose a calculation method that reflects road complexity comprehensively.

This paper concentrates on proposing a road-featurebased multiparameter road complexity calculation model of off-road environment. This model takes the longitudinal complexity, lateral complexity, and the travelable width of road into account, thus overcoming the drawbacks of a single indicator. With the multi-sensor-based data acquisition mobile platform, the road feature indicators like threedimensional scale, average slope, and adhesion characteristics of the travelable area are obtained. By using the improved AHP (Analytic Hierarchy Process) [21], the weights of indicators are determined. Finally, the proposed road complexity calculation model is verified by calculating complexities of several cross-country roads, and the quantitative analysis of the road complexity is realized. By testing on roads with different complexities, it is possible to assess the level of UGV and to facilitate quantitative evaluation of UGV.

\section{Complexity-Calculation-Based Off-Road Environmental Data Acquisition System}

2.1. Overall Architecture. In order to calculate the road complexity better, it is vital to have an accurate and comprehensive data acquisition of the road and its surface. The methods to acquire road environmental data can be divided into two major classes: the direct acquisition and the indirect acquisition. The direct acquisition method focuses on collecting the vertical road elevation data, while the indirect acquisition method obtains vibration response of vehicle to calculate TRI (truck ride index) [22], IRI, DLI, etc. Comparatively speaking, the indirect method can not obtain the real road surface data, while the direct acquisition method can obtain more complete road data, thus reflecting the road quality better.

In the past few decades, a number of methods have been proposed and experimented to obtain road data directly. Studies have shown that satellite images, aerial photographs, and point cloud data are main data sources for road information [23]. Satellite images and aerial photographs can provide road pixels and its two-dimensional (2D) location information [24], while accurate three-dimensional (3D) road information can only be obtained from point cloud data captured with mobile lidar system (MLS) [25]. MLS is gaining popularity in 3D mapping applications along various road corridors $[26,27]$. The precision and accuracy of road information obtained by MLS have been verified [28-30]. A few attempts have been made to compute the road geometry parameters from the MLS dataset. For the characterization of the vertical profiles and cross-sections of roads, a four-step method was devised using MLS data [31]. This method used a hierarchical strategy that includes segmentation, principal component analysis- (PCA-) based orthogonal regression, filtering, and parameter extraction procedures to process the MLS data and computed the best-fit geometric parameters of vertical and cross-sections. Aiming at the determination of road slopes, González-Jorge et al. used the different echoes of the lidar to perform automatic vegetation filtering and georeferenced points to avoid the GPS drift between different surveying [32]. In order to obtain high-precision road terrain, Yadav et al. proposed an automatic method to process MLS data [33]. This method used specific characteristics of a road to identify road surface points and refine road boundary [23]. Further road boundary was approximated to piecewise connected linear segments by using a best-fit polynomial. Road center points were computed by selecting collinear road boundary points at transverse direction. Finally the road width and road slope were computed by road center points and road boundary points.

In this paper, a complexity-calculation-based off-road environmental data acquisition system is established to obtain road data directly. The basic architecture of off-road environmental data acquisition system is a stage achievement, which combines the studies of UGS's environmental design based on complexity and the evaluation of UGS's autonomous capability. As shown in Figure 1, the basic architecture regards the calculation of complexity as the main line, collects local environmental data through cameras, lidars, and other sensors installed on the data acquisition equipment system, and then uses the vehicle-mounted and off-site test environmental data processing software system to extract parameters for complexity calculation. According to the combination method of test environmental elements and the complexity calculation method, along with the design of dynamic and static elements as well as task design in 


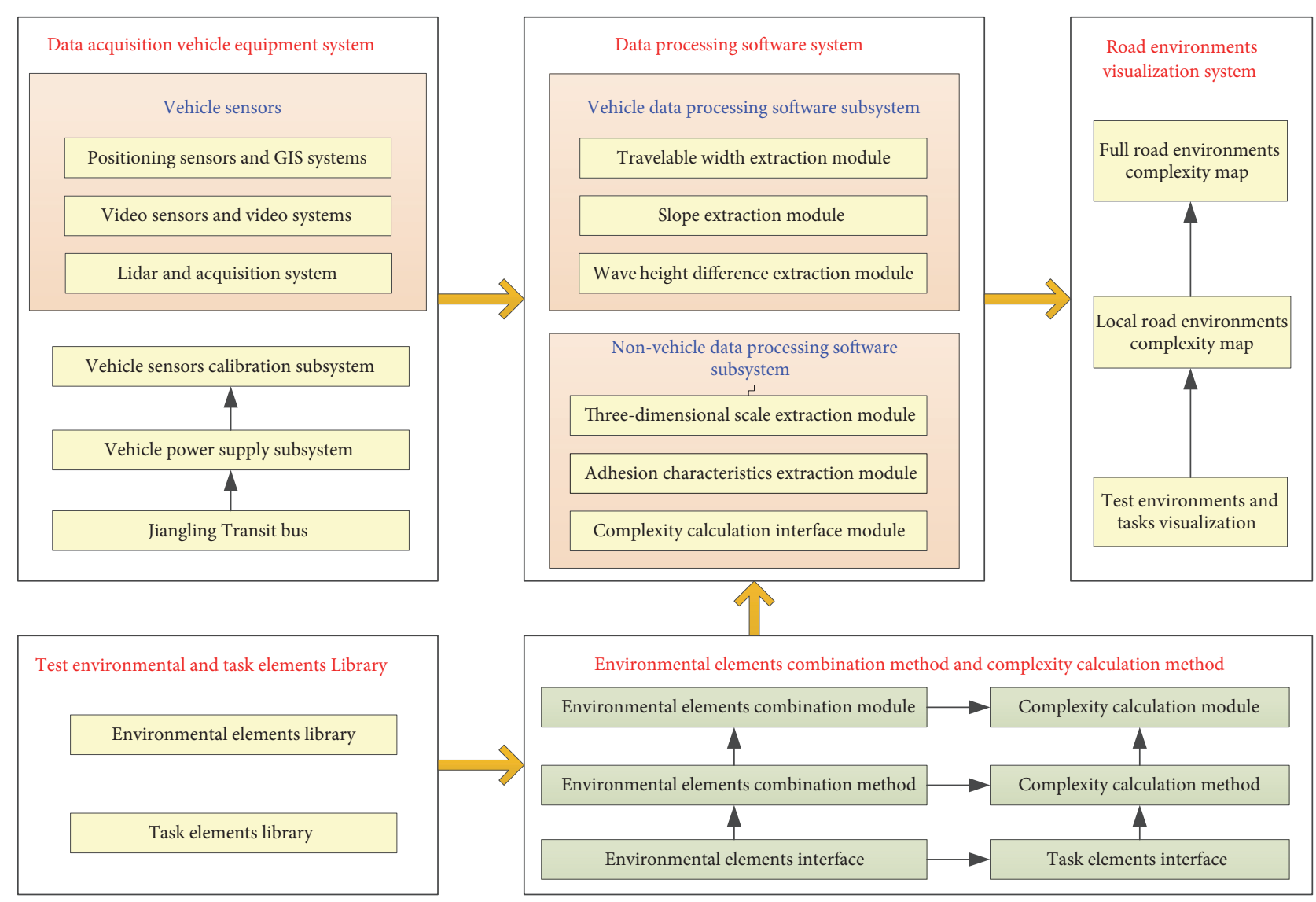

FIGURE 1: Basic architecture of off-road environmental data acquisition system.

battlefield environment, the full complexity map of roads is formed, which provides a scientific basis to further improve the rules and evaluation method of UGV's test.

2.2. Data Acquisition Mobile Platform. Data acquisition equipment system takes a Ford car as platform where the software and hardware system of the multisensor information collection is established. Data acquisition platform R\&D (research and development) framework shown in Figure 2 includes overall design of platform, selection and installation of sensors, calibration of sensors, and development of data acquisition software. A brief introduction is as follows.

The overall design of the platform is based on a Ford platform. The Ford car has larger interior space, so the necessary sensors, power supply systems (providing power for sensors and data acquisition system), and data storage systems are installed. The platform also has the synchronous acquisition software to achieve multisensor information synchronization acquisition.

Data acquisition sensors mainly include single-layer lidar, multilayer lidar, binocular camera, camera, GPS navigation equipment, gradiometer, and meteorological collection devices. Some sensors are installed on a platform fixed outside the roof; the others are installed inside the vehicle. The arrangement of sensors is shown in Figure 3. In this paper, sensor model of single-layer lidar is UTM-30LX-EW

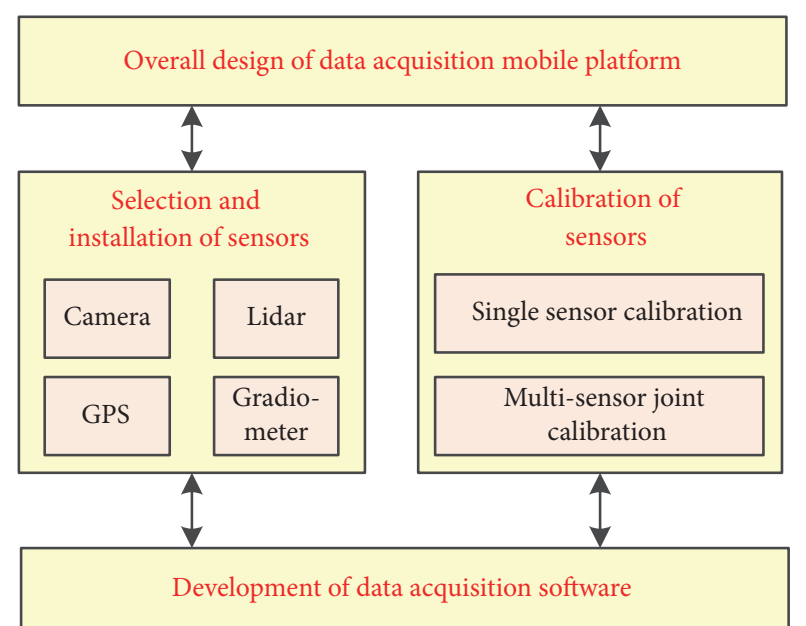

FIGURE 2: Data acquisition platform R\&D framework.

$2 \mathrm{D}$ laser scanner, and its sampling period is 0.10 s. GPS navigation equipment uses a Simpak982 GNSS (Global Navigation Satellite System) receiver to obtain location information of the path, and its sampling period is $0.05 \mathrm{~s}$. The resolution of the camera is $800 * 600$, and the sensor model of gradiometer is DP23850. Binocular camera is used to aid in ranging, and its data are not used in the experiment. 


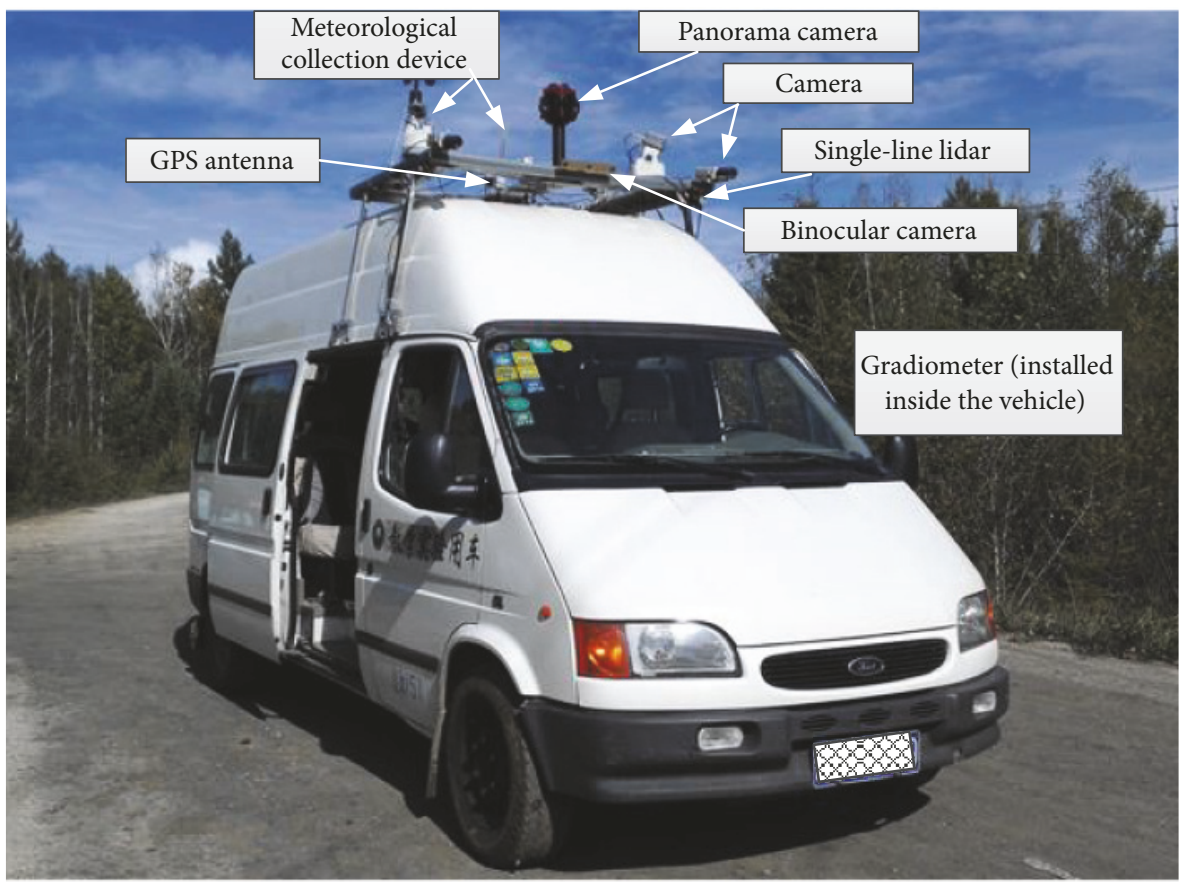

Figure 3: Arrangement of sensors.

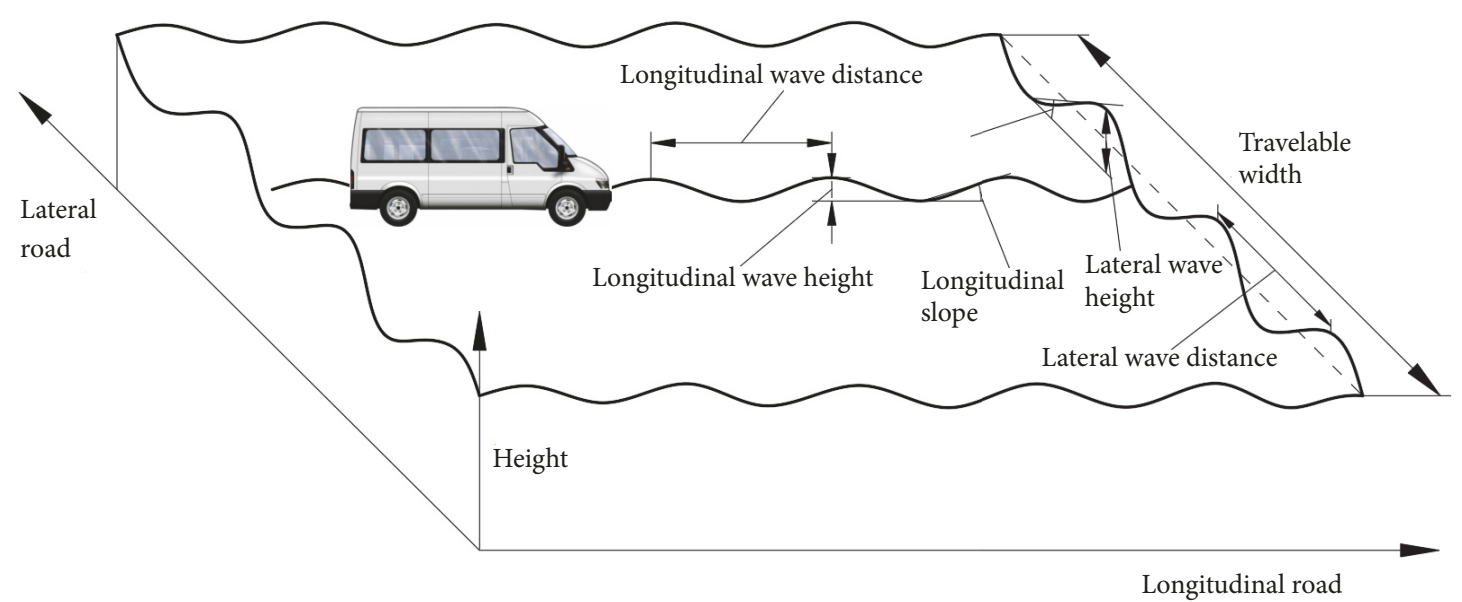

FIGURE 4: Off-road environment.

In order to ensure the sensors installed in different locations have a unified coordinate system, it is necessary to establish the corresponding relationships between different sensors. By setting calibration objects and manually selecting the corresponding points, the rotation matrixes and the translation vectors among different coordinate systems of sensors are solved. Based on the difference of sensors, the calibrations are divided into lidar calibration, camera calibration, GPS system calibration, wheel encoder calibration, and the joint calibration. For example, the lidar's installation parameters are pitch angle and roll angle, which are calibrated by using isosceles right triangle calibration plate and square calibration plate, respectively [34].

\section{Road-Feature-Based Road Complexity Calculation Model}

3.1. Establishment of Complexity Calculation Model. Road data can be obtained through data acquisition mobile platform. As shown in Figure 4, the road features can be divided into longitudinal features, lateral features, and travelable width features, wherein the longitudinal road features affect the longitudinal stability of vehicles and lateral road features affect vehicle lateral stability, while the travelable width affects vehicle passability directly. Compared with urban roads, the surface of cross-country roads has many gullies, and the existence of these gullies reduces the ride performance of 
vehicles. In this paper, we characterize cross-country roads by wave height difference, wave distance, slope, and adhesion characteristics, along with travelable width, and divide road complexity into longitudinal road complexity, lateral road complexity, and road width complexity. The road complexity $W_{d}$ can be calculated as

$$
W_{d}=W_{x} \cdot p_{1}+W_{y} \cdot p_{2}+W_{k} \cdot p_{3}
$$

The longitudinal road complexity $W_{x}$, lateral road complexity $W_{y}$, and road width complexity $W_{k}$ can be calculated as

$$
\begin{aligned}
& W_{x}=W_{x h d} \cdot q_{1}+W_{x \alpha} \cdot q_{2}+W_{x \varphi} \cdot q_{3} \\
& W_{y}=W_{y h d} \cdot r_{1}+W_{y \beta} \cdot r_{2}+W_{y \varphi} \cdot r_{3} \\
& W_{k}=\exp (-(K-B))
\end{aligned}
$$

where the road width limit $B$ normally is set to 2 , which characterizes the wheel track of the vehicle. $K$ is travelable width. $q_{1}, q_{2}, q_{3}, p_{1}, p_{2}, p_{3}, r_{1}, r_{2}, r_{3}$ are indicator weights.

The longitudinal road roughness $W_{\text {xhd }}$ includes longitudinal wave height difference complexity $W_{x h}$ and longitudinal wave distance complexity $W_{x d}$. $W_{x h d}$ can be solved as

$$
W_{x h d}=\sum_{i=0}^{4} m_{i}\left(\frac{W_{x h}^{i}+W_{x d}^{i}}{2}\right), \quad i=0,1 \cdots 4
$$

where

$$
\begin{aligned}
& W_{x h}^{i}=\frac{H_{x}^{i}}{H} \\
& W_{x d}^{i}=\operatorname{sech}\left|D_{x}^{i}-L\right|, \quad i=1,2,3,4 \\
& W_{x d}^{0}=W_{x d}^{1}
\end{aligned}
$$

It is to be noted that $H$ is a set value (the default value is $0.5 \mathrm{~m}$ ), which can be seen as the maximum depth of the gully which the vehicle can pass through. $m_{i}$ is an indicator weight. $H_{x}^{i}$ is the longitudinal wave height. $D_{x}^{i}$ is the longitudinal wave distance between the peak position and the maximum peak position in a sampling interval. $L$ is a constant with 3 , which characterizes the wheelbase of the vehicle.

The longitudinal slope complexity $W_{x \alpha}$ and longitudinal adhesion characteristics complexity $W_{x \varphi}$ are calculated by the following equations:

$$
\begin{aligned}
& W_{x \alpha}=\tan \alpha \\
& W_{x \varphi}=C \cdot \operatorname{sech}(2 \cdot \varphi)
\end{aligned}
$$

where $C$ is an influencing factor: when the wheels are on the road with different adhesion characteristics at the same time, the value of $C$ is 1.05; otherwise the value of $C$ is 1.0. $\alpha$ is the longitudinal slope and the unit is degree. $\varphi$ is the adhesion coefficient, and sech is a hyperbolic function.
By substituting (5)-(9) into (2), the longitudinal road complexity $W_{x}$ can be obtained. With the same method, the equations to calculate $W_{y}$ are

$$
\begin{aligned}
W_{y h d} & =\sum_{j=0}^{4} n_{j}\left(\frac{W_{y h}^{j}+W_{y d}^{j}}{2}\right), \quad j=0,1 \cdots 4 \\
W_{y h}^{j} & =\frac{H_{y}^{j}}{H} \\
W_{y d}^{j} & =\operatorname{sech}\left|D_{y}^{j}-B\right|, \quad j=1,2,3,4 \\
W_{y d}^{0} & =W_{y d}^{1} \\
W_{y \beta} & =\tan \beta \\
W_{y \varphi} & =C \cdot \operatorname{sech}(2 \cdot \varphi)
\end{aligned}
$$

where $W_{y h d}$ is the lateral road roughness. $W_{y h}$ is the lateral wave height difference complexity. $W_{y d}$ is the lateral wave distance complexity. $n_{i}$ is an indicator weight. $H_{y}^{j}$ is the lateral wave height. $D_{y}^{j}$ is the lateral wave distance between the peak position and the maximum peak position in a sampling interval. $W_{y \beta}$ is the lateral slope complexity. $W_{y \varphi}$ is the lateral adhesion characteristics complexity. $\beta$ is the lateral slope. By substituting (10)-(14) into (3), the lateral road complexity $W_{y}$ can be obtained. Finally the road complexity $W_{d}$ is calculated by substituting (2)-(4) into (1). The overall complexity calculation model can be expressed by the following block diagram (see Figure 5).

3.2. Determination of the Indicator Weights. In order to apply the complexity calculation model proposed in this paper, it is of great importance to determine the weight of each indicator. Several feasible methods have been put forward so far, such as AHP, information entropy theory [35], and improved AHP. The improved AHP uses a three-demarcation method to construct judgment matrix, overcoming the subjectivity and blindness of traditional AHP. An optimal transfer matrix model is also used by improved AHP, so the consistency check of judgment matrix is avoided. According to our previous method [21], the specific steps of improved AHP to calculate indicator weight are as follows.

Step 1 (establish the judgment matrix). With the improved AHP, the 3-scale judgment matrix is constructed:

$$
\mathrm{A}=\left[\begin{array}{ccc}
a_{11} & \cdots & a_{1 n} \\
\vdots & \ddots & \vdots \\
a_{n 1} & \cdots & a_{n n}
\end{array}\right]
$$

where

$$
a_{i j}= \begin{cases}1, & i \text { is more important than } j . \\ 0, & i \text { is equally important than } j . \\ -1, & i \text { is not as important as } j .\end{cases}
$$




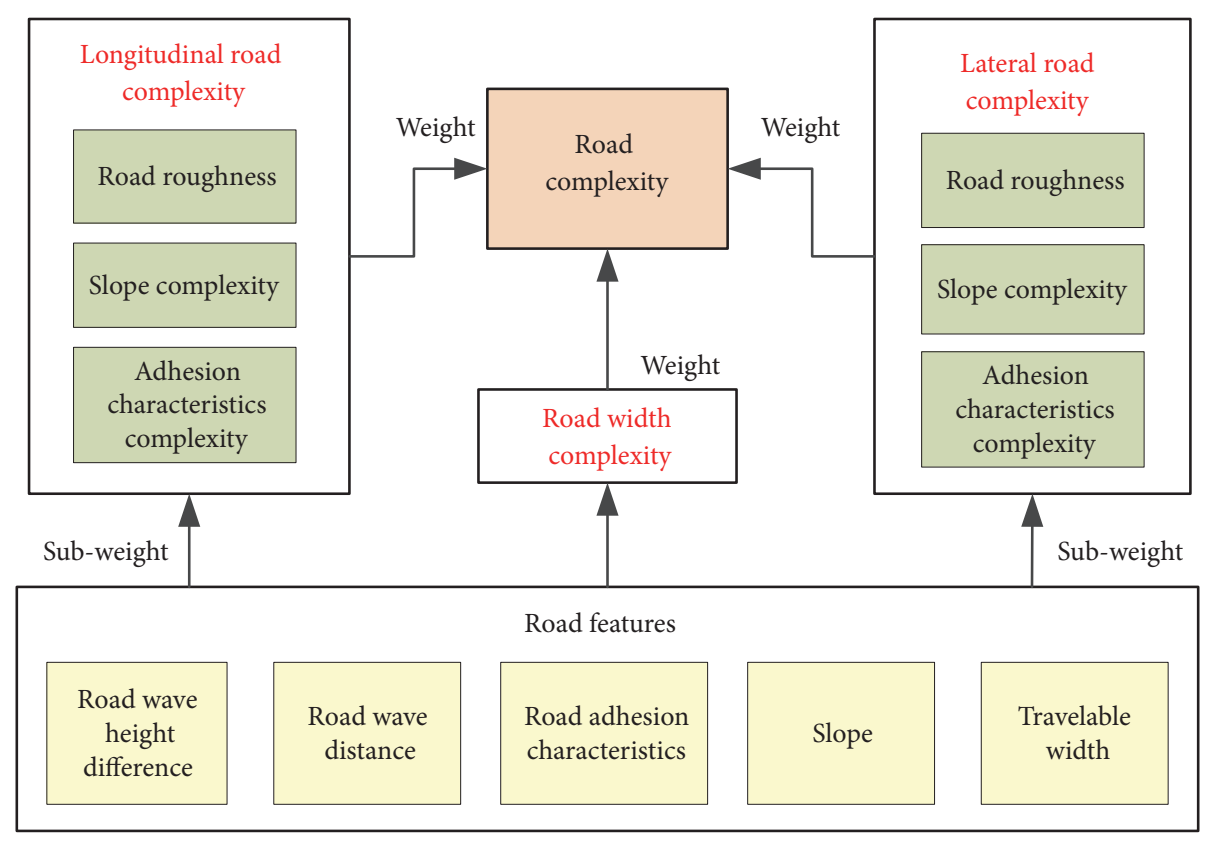

FIGURE 5: Complexity calculation model.

Step 2 (calculate the optimal transfer matrix). The optimal transfer matrix $\mathrm{R}$ is

$$
\mathrm{R}=\left[\begin{array}{ccc}
r_{11} & \cdots & r_{1 n} \\
\vdots & \ddots & \vdots \\
r_{n 1} & \cdots & r_{n n}
\end{array}\right]
$$

where $r_{i j}=(1 / n) \sum_{k=1}^{n}\left(a_{i k}+a_{k j}\right)$; then the optimal transfer matrix $\mathrm{R}$ is turned into the consistency matrix $\mathrm{D}$ :

$$
\mathrm{D}=\left[\begin{array}{ccc}
d_{11} & \cdots & d_{1 n} \\
\vdots & \ddots & \vdots \\
d_{n 1} & \cdots & d_{n n}
\end{array}\right]
$$

where $d_{i k}=\exp \left(r_{i k}\right)$.

Step 3 (calculate the relative indicator weights). The relative indicator weights can be represented by the eigenvector corresponding to the maximum eigenvalue in matrix $\mathrm{D}$. The eigenvector is calculated by product method:

$$
\begin{gathered}
\omega=\left[\omega_{1}, \omega_{2}, \cdots \omega_{n}\right]^{T} \\
\omega_{i}=\frac{\left(\prod_{k=1}^{n} d_{i k}\right)}{\sum_{k=1}^{n}\left(\prod_{k=1}^{n} d_{i k}\right)^{1 / n}}
\end{gathered}
$$

where $\omega=\left[\omega_{1}, \omega_{2}, \cdots \omega_{n}\right]^{T}$ is the weight vector of all factors.

\section{Experiments}

4.1. Test Sites. Off-road environment is complex and diverse and due to the limitation of test conditions, only a few crosscountry roads (see Figure 6) have been chosen, including muddy road, gully road, sand road, and hardened road, to calculate the road complexities. Test sites are in TaHe of China (52.2188N, 124.4124E), where the Unmanned Ground System Challenge (code-named Conquer Obstacle-2016) was held by the Chinese Army [11]. The total length is about 8 kilometers. Figure 7 shows part of the test route. See supplementary materials (available here) for a complete map.

4.2. Processing of Dataset. Off-road environmental data are obtained through data acquisition mobile platform. Simpak982 GNSS receiver obtains location information of the path, and the original data format is shown in Figure 8. Then GPS data are filtered to obtain latitude, longitude, altitude, and velocity. The angular resolution of UTM-30LX-EW 2D laser scanner is 0.25 degrees, and the scanning range is 120 degrees ahead. So there are 481 data points in each sampling period. The original data format of lidar is shown in Figure 9. It is to be noted that that there are many " 1 " in the data. During processing, these data will be replaced by their neighboring values. Then the data of lidar are processed with the following equation [34]:

$$
z=d_{l}^{k} \cdot \cos \left(b_{0}+k \cdot A\right) \cdot \sin \delta-z_{0}
$$

where $z$ is the road roughness, $d_{l}^{k}$ is the scanning distance between lidar and road surface, $k$ is the sequence number of lidar rays, $A$ is the lidar resolution, $b_{0}$ is the scanning starting angle, $\delta$ is the pitch angle, and $z_{0}$ is the road clearance of lidar. In transverse section of road, Y coordinate value is road roughness, and $\mathrm{X}$ coordinate value is calculated by the following formula:

$$
x=z_{0} \cdot \frac{\tan \left(b_{0}+k \cdot A\right)}{\sin \delta}
$$

The parameters like road wave height difference, wave distance, and travelable width are calculated with MATLAB. 


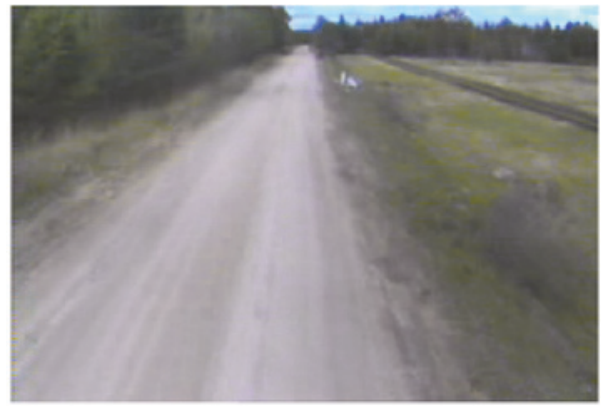

(a)

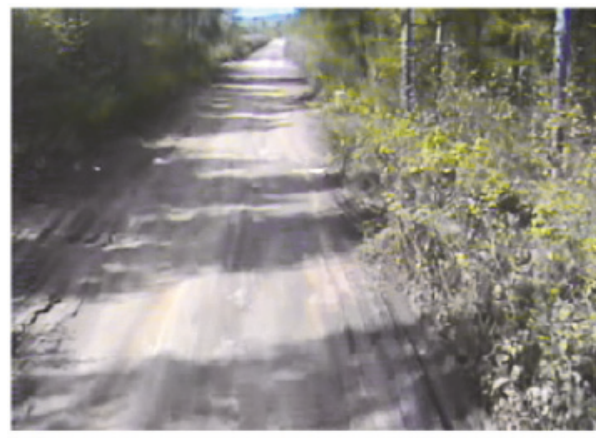

(c)

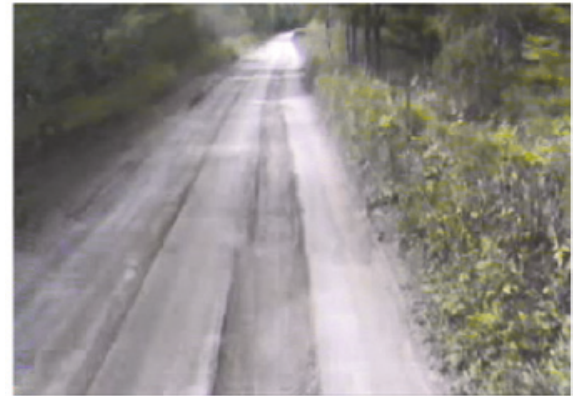

(b)

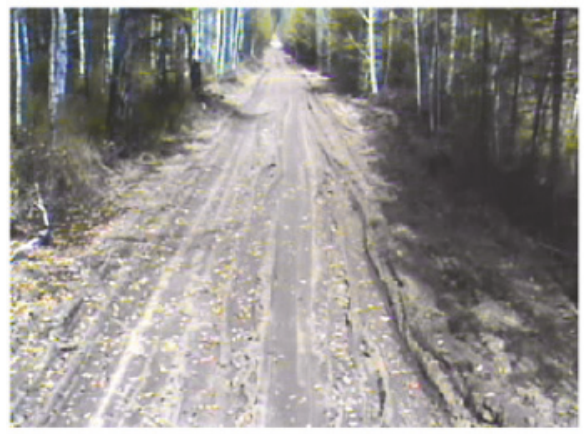

(d)

Figure 6: Cross-country roads: (a) dry unpaved road, (b) dry unpaved road with small gully, (c) dry unpaved road with larger gully, and (d) wet unpaved road with larger gully.

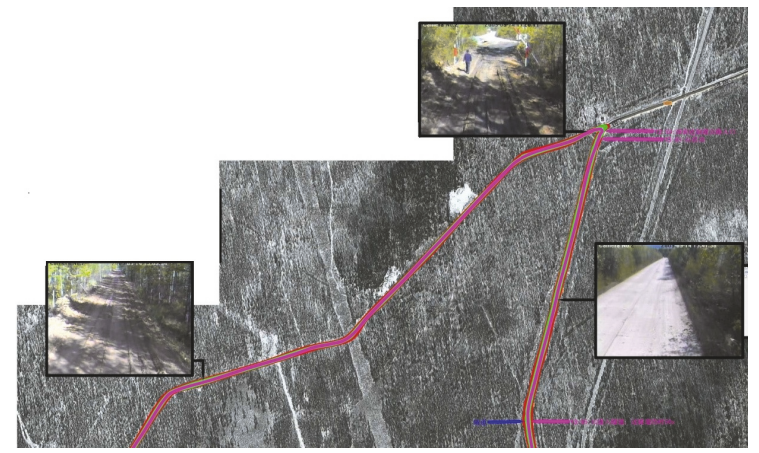

FIgURE 7: Test sites. The pink line is test route, and next to the route is road information displayed by the image.

In order to filter out small height changes, the MATLAB function $\operatorname{smooth}()$ is used to smooth data. The identification of peak positions is achieved by calling the differential function $\operatorname{diff}()$ twice. Then the wave height difference and wave distance can be calculated. Lateral road roughness is calculated directly from the lidar data collected in each sampling period, while longitudinal road roughness is calculated by combining GPS data and lidar data. In longitudinal section of road, Y coordinate value is the 241st value of lidar data in each sampling period, and $\mathrm{X}$ coordinate value is calculated by multiplying velocity by time. Velocity is extracted from GPS data, and time is the sampling period of UTM-30LXEW 2D laser scanner. The $\mathrm{X}$ coordinate value in longitudinal section of road is accumulated. The first coordinate value $X_{1}$ is zero, $X_{i}=X_{i-1}+V \cdot T$ ( $V$ is velocity, $T$ is time, and $i=2,3, \cdots \mathrm{N}$ ). When $X_{N} \geq L$, calculate longitudinal road complexity and zero the $\mathrm{X}$ coordinate value. At this point, $\mathrm{N}$ lateral road complexity has been calculated and the average is calculated to bring in (1). $L$ is a set value, referring to the classification of pavement structure in PIARC (Permanent International Association of Road Congresses). PIARC sets the construction wavelength of the uneven road to $0.5-50$ meters. In this paper, $L=25$ meters is chosen as a sampling length.

The calculation of the road width is achieved by comparing the height difference between two adjacent waves, and if the height difference is greater than threshold, the road boundary is considered to be reached. In this paper, the threshold is 0.5 meters. For detailed codes, see supplementary material. Figure 10 shows the process to identify the peak positions, and the largest five crest positions as well as the travelable width are marked in Figure 11.

In this paper, the slope measured on vehicle is approximately the slope of road. The slope data are obtained by gradiometer. Two gradiometers are installed on the floor of the copilot to measure longitudinal and lateral road slope, wherein one gradiometer is mounted along the direction of travel, and the other is mounted perpendicular to the direction of travel. Quantitative analysis of the adhesion coefficient is very difficult, so the adhesion coefficient of road surface is artificially set by comparing the real road conditions with the experience value. The real road condition is obtained by observing the video, and the experience value is selected by Table 1 . 
BGPGGA, 055428. 40, 5222. 8813632, N, 12441. 24968507, E, 2, 08, 1. 2, 422. 366, III, 4. 358, II, 7. 4, 0129*70 \$GPVTG, 175. 11, T, $-464.79, \mathbb{I I}, 4.54, \mathrm{~N}, 8.41, \mathrm{~K}, \mathrm{D} * 08$

\$GPRIC, 055428. 40, A, 5222. 88136372, N, 12441. 24968507, E, 4. 542, 175. 115, 140916, 999. 9000, E, D*04 \$GPGGA, 055428. 50, 5222. 88124385, N, 12441. 24969608, E, 2, 08, 1. 2, 422. 361, M, 4. 358, M. 7. 4, 0129*70 \$GPVTG, 176. 78, T, -463.12 , II, 4. 33, N, 8. 02, K, D*08

\$GPRIC, 055428. 50, A, 5222. 88124385, N, 12441. 24969608, E, 4. 329, 176. 781, 140916, 999. 9000, E, D*00 \$GPGGA, 055428. 60, 5222. 88111907, N, 12441. 24970171, E, 2, 08, 1. 2, 422. 361, M, 4. 358, M, 2. 6, 0129*73 \$GPVTG, 175. 57, T, -464. 33, II, 4. 34, N, 8. 03, K, D*04

\$GPRIC, 055428. 60, A, 5222. $88111907, \mathrm{~N}, 12441.24970171, \mathrm{E}, 4.338,175.572,140916,999.9000$, E, D*09

FIGURE 8: Original data format of GPS.

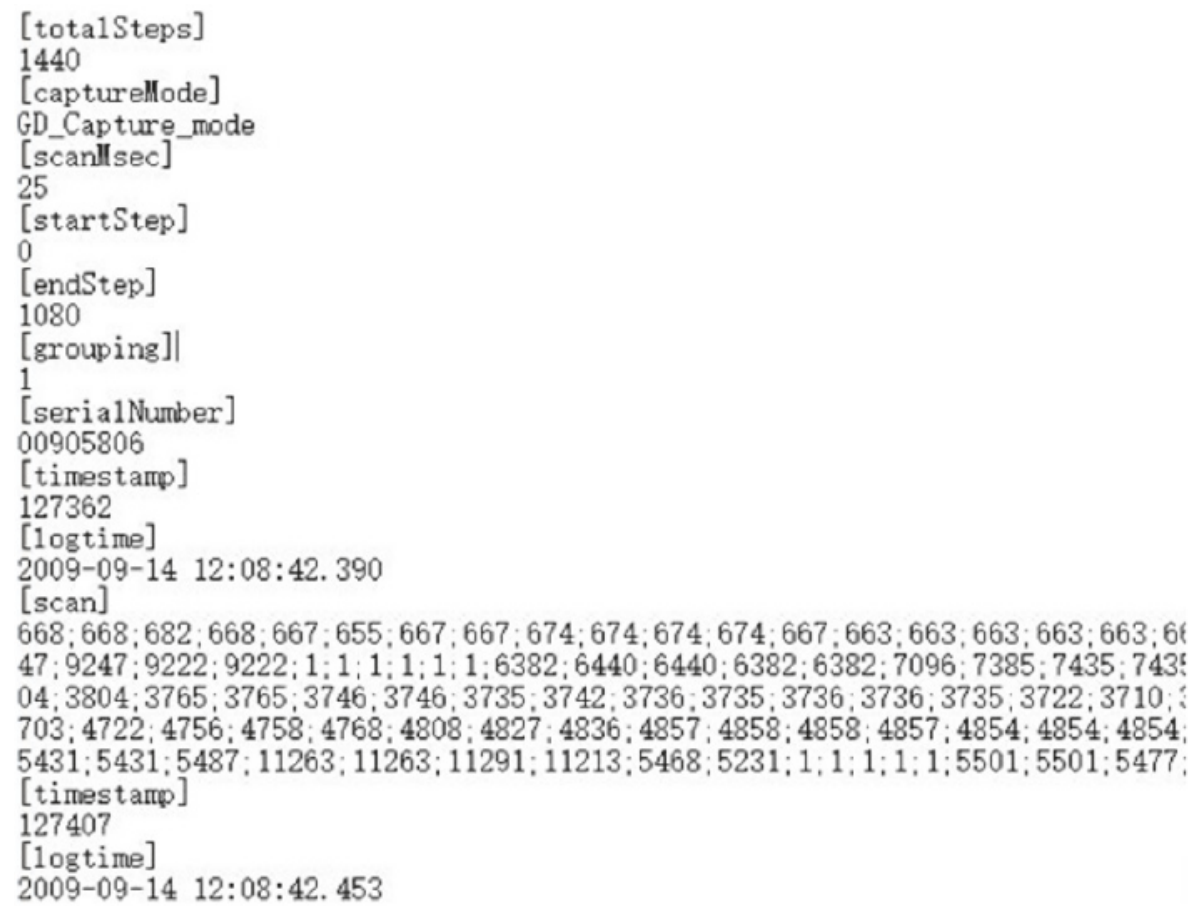

FIGURE 9: Original data format of lidar. The data are distances between lidar and road surface.

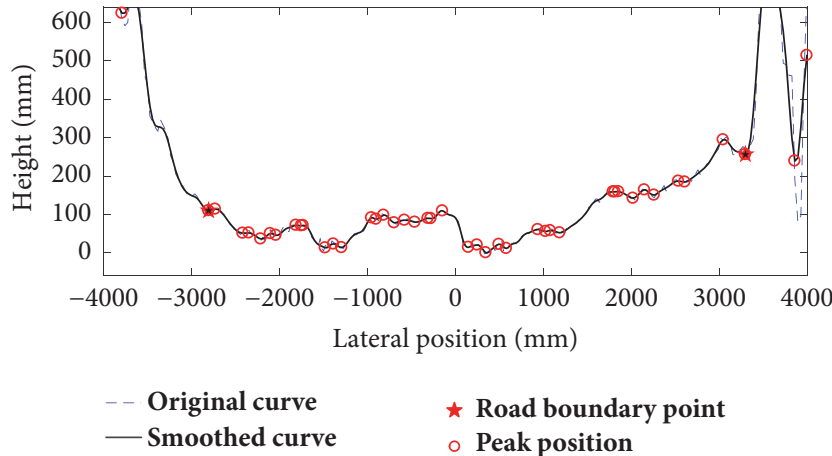

FIGURE 10: Identification of peak positions.

4.3. Results and Discussions. Table 2 shows the parameters of Figure 6, where the units of height and length are millimeters, and the units of slope are degrees. As mentioned in the previous section, road complexity calculation model includes longitudinal adhesion characteristics complexity, longitudinal road roughness, longitudinal slope complexity, lateral

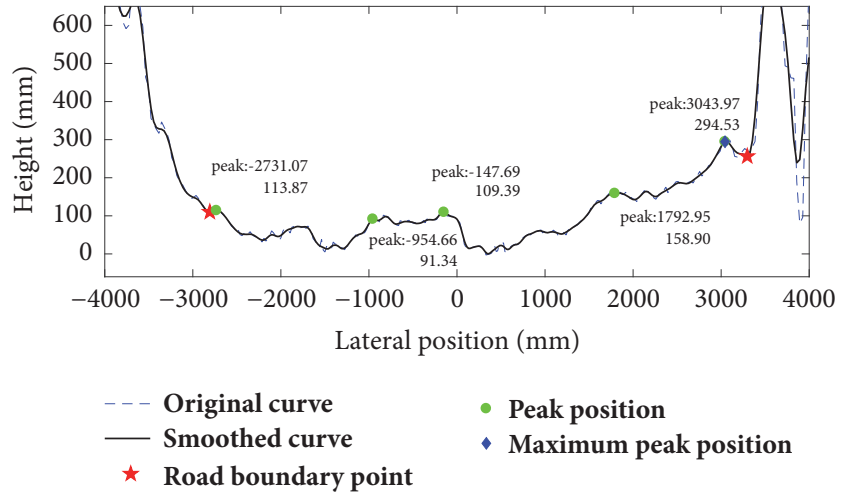

FIGURE 11: Largest five crest positions and travelable width.

adhesion characteristics complexity, lateral road roughness, lateral slope complexity, and road width complexity. So the road indicators are longitudinal adhesion characteristics, longitudinal road roughness, longitudinal slope, lateral adhesion characteristics, lateral road roughness, lateral slope, and travelable width. According to (15), (17), (18), and (19), matrix 
TABLE 1: Average adhesion coefficient on various road surfaces.

Road surfaces

Peak adhesion coefficient

Sliding adhesion coefficient

Asphalt or concrete road (dry)

$0.8 \sim 0.9$

0.75

Asphalt road (wet)

$0.5 \sim 0.7$

$0.45 \sim 0.60$

Concrete road (wet)

0.8

0.7

Gravel road

0.6

0.55

Dirt road (dry)

0.68

0.65

Dirt road (wet)

0.55

$0.4 \sim 0.5$

Snowy road

Ice road

0.1

0.07

TABle 2: Parameters of Figure 6.

\begin{tabular}{|c|c|c|c|c|}
\hline \multirow{2}{*}{ Features } & \multicolumn{4}{|c|}{ Parameters of road feature } \\
\hline & Figure 6(a) & Figure $6(b)$ & Figure 6(c) & Figure $6(d)$ \\
\hline$\overline{H_{y}^{0}}$ & 133.91 & 109.97 & 379.22 & 496.87 \\
\hline$H_{y}{ }^{1}$ & 29.387 & 106.72 & 327.03 & 340.62 \\
\hline$H_{y}^{2}$ & 24.813 & 94.947 & 139.56 & 340.06 \\
\hline$H_{y}^{3}$ & 22.686 & 77.712 & 121.41 & 254.11 \\
\hline$H_{y}^{4}$ & 22.067 & 62.637 & 109.57 & 163.80 \\
\hline$H_{x}^{0}$ & 24.791 & 76.431 & 126.59 & 13.856 \\
\hline$H_{x}^{1}$ & 24.783 & 50.215 & 117.59 & 10.416 \\
\hline$H_{x}^{2}$ & 19.426 & 40.663 & 112.83 & 10.319 \\
\hline$H_{x}^{3}$ & 16.336 & 38.407 & 98.155 & 10.205 \\
\hline$H_{x}^{4}$ & 15.913 & 31.081 & 63.261 & 8.8541 \\
\hline$D_{y}^{0}$ & 237.00 & 1251.0 & 233.22 & 1067.7 \\
\hline$D_{y}^{1}$ & 237.00 & 1251.0 & 233.22 & 1067.7 \\
\hline$D_{y}^{2}$ & 3605.7 & 3191.7 & 1720.7 & 5823.9 \\
\hline$D_{y}^{3}$ & 5438.7 & 3998.6 & 8059.6 & 1704.9 \\
\hline$D_{y}^{4}$ & 2313.3 & 5775.1 & 587.14 & 616.60 \\
\hline$D_{x}^{0}$ & 7900 & 1120 & 7960 & 10195 \\
\hline$D_{x}^{1}$ & 7900 & 1120 & 7960 & 10195 \\
\hline$D_{x}^{2}$ & 3900 & 4090 & 2010 & 10903 \\
\hline$D_{x}^{3}$ & 5130 & 3270 & 3370 & 4200.6 \\
\hline$D_{x}^{4}$ & 2770 & 1790 & 4300 & 1510.3 \\
\hline$\alpha$ & 1.25 & 1.23 & 2.45 & 1.95 \\
\hline$\beta$ & 0.30 & 0.75 & 1.05 & 1.25 \\
\hline$\varphi$ & 0.60 & 0.60 & 0.60 & 0.40 \\
\hline K & 6576 & 6103 & 8899 & 8229 \\
\hline
\end{tabular}

A, optimal transfer matrix $\mathrm{R}$, matrix $\mathrm{D}$, and the indicator weights $\omega$ are as follows:

$$
A=\left[\begin{array}{ccccccc}
0 & 1 & 1 & -1 & 1 & 1 & 1 \\
-1 & 0 & 1 & -1 & -1 & 1 & 1 \\
-1 & -1 & 0 & -1 & -1 & -1 & 1 \\
1 & 1 & 1 & 0 & 1 & 1 & 1 \\
-1 & 1 & 1 & -1 & 0 & 1 & 1 \\
-1 & -1 & 1 & -1 & -1 & 0 & 1 \\
-1 & -1 & -1 & -1 & -1 & -1 & 0
\end{array}\right]
$$

TABLE 3: Weights.

\begin{tabular}{lccccc}
\hline Symbols & $\boldsymbol{m}_{\mathbf{0}}$ & $\boldsymbol{m}_{\mathbf{1}}$ & $\boldsymbol{m}_{\mathbf{2}}$ & $\boldsymbol{m}_{\mathbf{3}}$ & $\boldsymbol{m}_{\mathbf{4}}$ \\
\hline values & 0.3 & 0.3 & 0.2 & 0.1 & 0.1 \\
\hline Symbols & $n_{0}$ & $n_{1}$ & $n_{2}$ & $n_{3}$ & $n_{4}$ \\
\hline values & 0.3 & 0.3 & 0.2 & 0.1 & 0.1 \\
\hline Symbols & $p_{1}$ & $p_{2}$ & $p_{3}$ & $q_{1}$ & $q_{2}$ \\
\hline values & 0.407 & 0.542 & 0.052 & 0.300 & 0.169 \\
\hline Symbols & $q_{3}$ & $r_{1}$ & $r_{2}$ & $r_{3}$ & $C$ \\
\hline values & 0.531 & 0.299 & 0.170 & 0.531 & 1.0 \\
\hline
\end{tabular}

$$
\begin{aligned}
\mathrm{R} & =\frac{1}{7}\left[\begin{array}{ccccccc}
0 & 4 & 8 & -2 & 2 & 6 & 10 \\
-4 & 0 & 4 & -6 & -2 & 2 & 6 \\
-8 & -4 & 0 & -10 & -6 & -2 & 2 \\
2 & 6 & 10 & 0 & 4 & 8 & 12 \\
-2 & 2 & 6 & -4 & 0 & 4 & 8 \\
-6 & -2 & 2 & -8 & -4 & 0 & 4 \\
-10 & -6 & -2 & -12 & -8 & -4 & 0
\end{array}\right] \\
\mathrm{D} & =\left[\begin{array}{cccccccc}
1 & 1.77 & 3.14 & 0.75 & 1.33 & 2.36 & 4.17 \\
0.56 & 1 & 1.77 & 0.42 & 0.75 & 1.33 & 2.36 \\
0.32 & 0.56 & 1 & 0.24 & 0.42 & 0.75 & 1.33 \\
1.33 & 2.36 & 4.17 & 1 & 1.77 & 3.14 & 5.55 \\
0.75 & 1.33 & 2.36 & 0.56 & 1 & 1.77 & 3.14 \\
0.42 & 0.75 & 1.33 & 0.32 & 0.56 & 1 & 1.77 \\
0.24 & 0.42 & 0.75 & 0.18 & 0.32 & 0.56 & 1
\end{array}\right]
\end{aligned}
$$

$\omega$

$$
=\left[\begin{array}{lllllll}
0.216 & 0.122 & 0.069 & 0.288 & 0.162 & 0.092 & 0.052
\end{array}\right]^{T}
$$

The final weights used in road complexity calculation model are shown in Table 3 and the complexity calculation results are shown in Table 4.

As can be seen from Table 2, the lateral wave height difference is bigger than the longitudinal wave height difference, so the lateral complexity is also larger than the longitudinal 
TABLE 4: Results.

\begin{tabular}{lcccc}
\hline \multirow{2}{*}{ Symbols } & \multicolumn{4}{c}{ Complexity calculation results } \\
& Figure 6(a) & Figure 6(b) & Figure 6(c) & Figure 6(d) \\
\hline $\boldsymbol{W}_{\boldsymbol{y} \boldsymbol{h d}}$ & 0.248 & 0.401 & 0.482 & 0.641 \\
$\boldsymbol{W}_{\boldsymbol{x} \boldsymbol{h} \boldsymbol{d}}$ & 0.022 & 0.279 & 0.253 & 0.061 \\
$\boldsymbol{W}_{\boldsymbol{y} \boldsymbol{\beta}}$ & 0.005 & 0.013 & 0.018 & 0.022 \\
$\boldsymbol{W}_{\boldsymbol{x} \boldsymbol{\alpha}}$ & 0.022 & 0.022 & 0.043 & 0.034 \\
$\boldsymbol{W}_{\boldsymbol{y} \boldsymbol{}}$ & 0.552 & 0.552 & 0.552 & 0.748 \\
$\boldsymbol{W}_{\boldsymbol{x} \varphi}$ & 0.552 & 0.552 & 0.552 & 0.748 \\
$W_{x}$ & 0.304 & 0.381 & 0.377 & 0.421 \\
$W_{y}$ & 0.358 & 0.406 & 0.431 & 0.579 \\
$W_{k}$ & 0.010 & 0.017 & 0.001 & 0.002 \\
$W_{d}$ & 0.318 & 0.376 & 0.387 & 0.485 \\
\hline
\end{tabular}

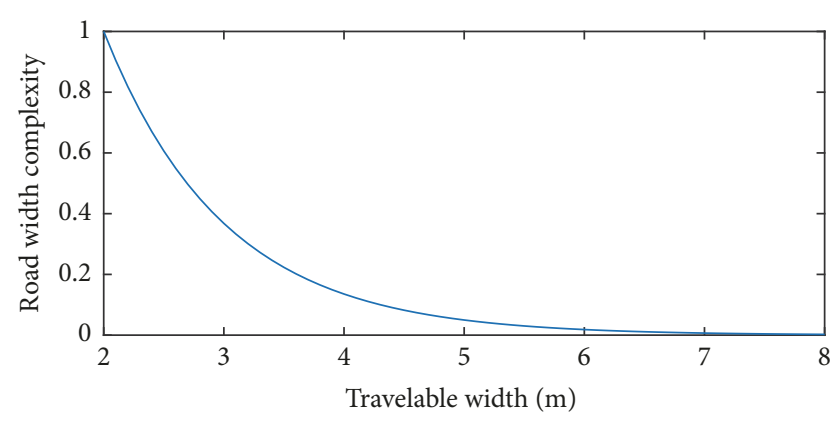

FIGURE 12: Relation curve between complexity and travelable width.

complexity. Table 4 shows that the complexities of crosscountry roads (Figure 6) increase in turn, and it is in line with the reality. The main influence factor of road complexity is the lateral complexity, which has impacts on lateral stability of the vehicle. The influence of road width on complexity is not obvious, since the travelable width obviously allows vehicle to pass smoothly. When travelable width becomes smaller, the complexity increases exponentially. The relation curve between road complexity and travelable width is shown in Figure 12. The influence of the adhesion coefficient can be seen from the parameters of Figures 6(c) and 6(d). The main difference between Figures 6(c) and 6(d) is that the adhesion coefficient is different. The adhesion coefficient of Figure 6(c) is 0.6 , while the adhesion coefficient of Figure 6(d) is 0.4. So the road complexity values are 0.387 and 0.485 , respectively, with a difference of about 0.1. By setting different adhesion coefficients for Figure 6(d), the relation curve between the road complexity and adhesion coefficient can be obtained. As it can be seen from Figure 13, for the same road, with the decrease of adhesion coefficient, road complexity increases linearly.

From the results and analysis above, it is shown that the complexity of the road can be quantitatively characterized by road complexity. Road complexity reflects road accessibility. In fact, the test sites of this paper is set for the Unmanned Ground System Challenge. By setting road environment with different complexity, the level of the unmanned vehicle can be checked. During the competition, the road environment is

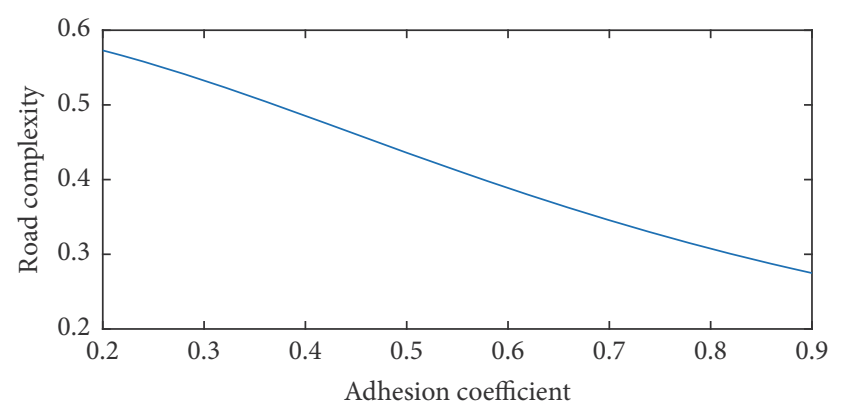

FIGURE 13: Relation curve between complexity and adhesion coefficient.

set to dry unpaved road, wet unpaved road, gully road, and paved road, and the road adhesion coefficient is in the range of 0.4-0.8. Only the UGV with excellent off-road capability can successfully pass all the test sections. By setting up snow and ice roads or muddy roads, it is possible to set up a more complex road environment to test the adaptability of UGV in harsh environments.

\section{Conclusions}

In this paper, a road-feature-based multiparameter road complexity calculation model of off-road environment is presented. This model uses wave height difference, wave distance, slope, adhesion characteristics, and travelable width as road feature indicators and takes the longitudinal road complexity, lateral road complexity, and the travelable width into account, thus overcoming the drawbacks of single indicator of other methods, reflecting the complexities of different roads more comprehensively. Through the calculation of road complexities in cross-country roads, the feasibility of this model is verified. Experiments show that this model can quantitatively analyze road complexity in off-road environment and provide a theoretical support for the scientific calculation of different road complexities.

In addition, an overall architecture of data acquisition system is also presented, which provides an effective method for environmental data acquisition. The indicator weights are calculated by using improved AHP, which makes the determination of indicator weights more scientific.

Due to the limited test samples, only some road complexities are calculated. In future work, more tests are expected to be conducted to obtain more road environmental data, and a road complexity database is expected to be established to provide data support for environment design of unmanned vehicle testing.

\section{Data Availability}

For the data and code used in this paper, see supplementary materials.

\section{Conflicts of Interest}

The authors declare that there are no conflicts of interest regarding the publication of this paper. 


\section{Acknowledgments}

The authors thank all the participants for their kind cooperation. This research is supported by the National Natural Science Foundation of China (no. 61271376 and no. 61272509), the National Key R\&D Program of China (no. 2017YFC0804803 and no. 2017YFC0804808), and the Research Foundation of Shanghai International Automobile City (no. 20160341208).

\section{Supplementary Materials}

See supplementary material for the road roughness data, data processing, and complexity calculation code. (Supplementary Materials)

\section{References}

[1] X. Guangming, L. Gao, S. Wu, and Y. Zhao, Intelligent Behaviors and Test and Evaluation for Unmanned Ground Vehicle, Beijing Institute of Technology Press, Beijing, China, 2015.

[2] X. Gao, Research on Information Processing of Intelligent Behavior and Evaluation of Unmanned Vehicle Based on Typical Working Condition, Beijing Institute of Technology, Beijing, China, 2012.

[3] S. Yang, Quantitative Evaluation of Intelligence Levels for Unmanned Ground Vehicles, Beijing Institute of Technology, Beijing, China, 2014.

[4] C. M. Martinez, X. Hu, D. Cao, E. Velenis, B. Gao, and M. Wellers, "Energy management in plug-in hybrid electric vehicles: recent progress and a connected vehicles perspective," IEEE Transactions on Vehicular Technology, vol. 66, no. 6, pp. 4534-4549, 2017.

[5] Y. Sun and H.-Y. Chen, "Research on test and evaluation of unmanned ground vehicles," Acta Armamentarii, vol. 36, no. 6, pp. 978-986, 2015.

[6] R. Behringer, S. Sundareswaran, R. Daily et al., "The DARPA grand challenge - development of an autonomous vehicle," in Proceedings of the IEEE Intelligent Vehicles Symposium, 2004, pp. 226-231, Parma, Italy.

[7] I. Miller, S. Lupashin, N. Zych et al., "Cornell University's 2005 DARPA Grand Challenge entry," Journal of Field Robotics, vol. 23, no. 8, pp. 625-652, 2006.

[8] C. Urmson, J. Anhah, D. Bagnell et al., "Autonomous driving in urban environments: Boss and the urban challenge," Journal of Field Robotics, vol. 25, no. 8, pp. 425-466, 2008.

[9] G. Xiong, P. Zhou, S. Zhou et al., "Autonomous driving of intelligent vehicle BIT in 2009 future challenge of China," in Proceedings of the 2010 IEEE Intelligent Vehicles Symposium, IV 2010, pp. 1049-1053, San Diego, Calif, USA, June 2010.

[10] "Future Challenge 2012," http://www.gov.cn/gzdt/2012-10/31/ content_2255142.html.

[11] “The Unmanned Ground System Challenge," http://eng.mod .gov.cn/TopNews/2016-10/19/content_4749636.htm.

[12] X. Hu, H. Wang, and X. Tang, "Cyber-Physical Control for Energy-Saving Vehicle Following with Connectivity," IEEE Transactions on Industrial Electronics, vol. 64, no. 11, pp. 85788587, 2017.

[13] ASTM, "Standard terminology relating to vehicle- pavement systems," Tech. Rep. ASTM 2012 E867, American Society for Testing Materials, ASTM, Pennsylvania, Pa, USA, 2012.
[14] Z. Cunwei and R. Chen, "Quantitative analysis and establishment of grade evaluation index of road unevenness," Northern Communications, vol. 78, no. 8, pp. 69-72, 2016.

[15] ASTM, "Standard practice for computing international roughness index of roads from longitudinal profile measurements," Tech. Rep. ASTM 2008 E1926, American Society for Testing Materials, ASTM, Pennsylvania, Pa, USA, 2008.

[16] M. W. Sayers, "On the calculation of international roughness index from longitudinal road profile," Transportation Research Record, no. 1501, pp. 1-12, 1995.

[17] J. Marcondes, G. J. Burgess, R. Harichandran, and M. B. Snyder, "Spectral analysis of highway pavement roughness," Journal of Transportation Engineering, vol. 117, no. 5, pp. 540-549, 1991.

[18] IS0, "Mechanical vibration-road surface profiles-reporting of measured data," Tech. Rep. IS0 8608:1995, ISO/TC108/SC 2, International Organization for Standardization, Geneve, Switzerland, 1995.

[19] K. Chatti and D. Lee, "Development of new profile-based truck dynamic load index," Transportation Research Record, no. 1806, pp. 149-159, 2002.

[20] P. Múčka, "Current approaches to quantify the longitudinal road roughness," International Journal of Pavement Engineering, vol. 17, no. 8, pp. 659-679, 2016.

[21] F. Dong, Z. Yanan, and L. Gao, "Application of gray correlation and improved AHP to evaluation on intelligent U-Turn behavior of unmanned vehicles," in Proceedings of the 2015 8th International Symposium on Computational Intelligence and Design, ISCID 2015, vol. 12, pp. 25-29, 2015.

[22] H. Prem, E. Ramsay, and J. McLean, "A road profile based truck ride index (TRI)," in Proceedings of the 6th International Symposium on Heavy Vehicle Weights and Dimensions, pp. 483505, Saskatoon, Saskatchewan, Canada, 2000.

[23] M. Yadav, A. K. Singh, and B. Lohani, "Extraction of road surface from mobile LiDAR data of complex road environment," International Journal of Remote Sensing, vol. 38, no. 16, pp. 4645-4672, 2017.

[24] M. Mokhtarzade and M. J. V. Zoej, "Road detection from highresolution satellite images using artificial neural networks," International Journal of Applied Earth Observation and Geoinformation, vol. 9, no. 1, pp. 32-40, 2007.

[25] M. Yadav, S. Goel, A. K. Singh, and B. Lohani, "Developing basic design and mathematical framework for a mobile mapping system-a case study using available sensors," Journal of the Indian Society of Remote Sensing, vol. 42, no. 2, pp. 301-310, 2014.

[26] M. Yadav and A. K. Singh, "Rural road surface extraction using mobile LiDAR point cloud data," Journal of the Indian Society of Remote Sensing, vol. 46, no. 4, pp. 531-538, 2018.

[27] C. K. Toth, "R\&D of mobile lidar mapping and future trends," in Proceedings of the ASPRS 2009 Annual Conference, Baltimore, Md, USA, 2009.

[28] D. Barber, J. Mills, and S. Smith-Voysey, "Geometric validation of a ground-based mobile laser scanning system," ISPRS Journal of Photogrammetry and Remote Sensing, vol. 63, no. 1, pp. 128141,2008

[29] N. Haala, M. Peter, A. Cefalu, and J. Kremer, "Mobile lidar mapping for urban data capture," in Proceedings of the 14th International Conference on Virtual Systems and Multimedia, Limassol, Cyprus, 2008.

[30] K. Williams, M. J. Olsen, G. V. Roe, and C. Glennie, "Synthesis of transportation applications of mobile LIDAR," Remote Sensing, vol. 5, no. 9, pp. 4652-4692, 2013. 
[31] A. Holgado-Barco, D. Gonzalez-Aguilera, P. Arias-Sanchez, and J. Martinez-Sanchez, "An automated approach to vertical road characterisation using mobile LiDAR systems: Longitudinal profiles and cross-sections," ISPRS Journal of Photogrammetry and Remote Sensing, vol. 96, pp. 28-37, 2014.

[32] H. González-Jorge, P. Arias Sanchez, I. Puente, and J. Martínez, "Surveying of road slopes using mobile LiDAR," Gerontechnology, vol. 11, no. 2, 2012.

[33] M. Yadav, A. K. Singh, and B. Lohani, "Computation of road geometry parameters using mobile LiDAR system," Remote Sensing Applications: Society and Environment, vol. 10, pp. 1823, 2018.

[34] C. Huiyan, G. Xiong, J. Gong, and Y. Jiang, Introduction to Self-Driving Car, Beijing Institute of Technology Press, Beijing, China, 1st edition, 2014.

[35] Y.-N. Zhao, K.-W. Meng, and L. Gao, "The entropy-cost function evaluation method for unmanned ground vehicles," Mathematical Problems in Engineering, vol. 2015, Article ID 410796, 6 pages, 2015. 


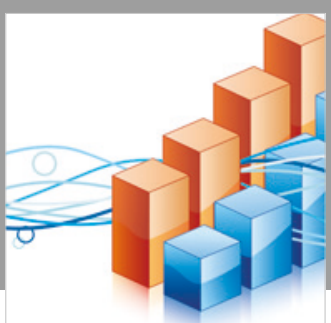

Advances in

Operations Research

\section{-n-m}
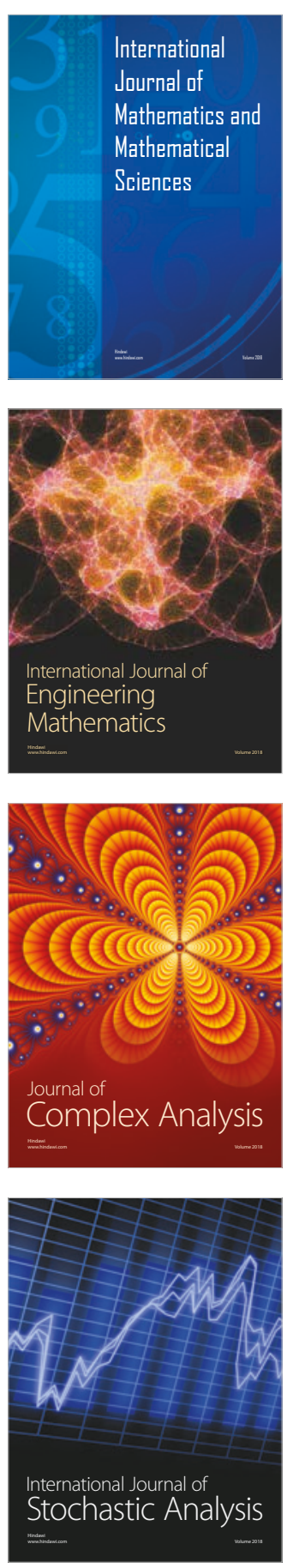
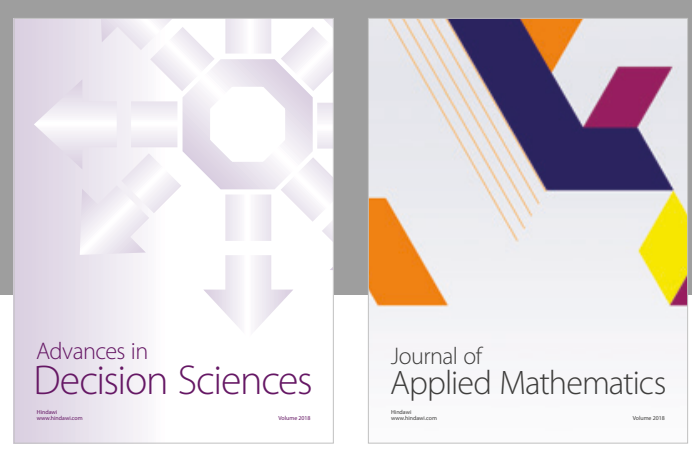

Journal of

Applied Mathematics
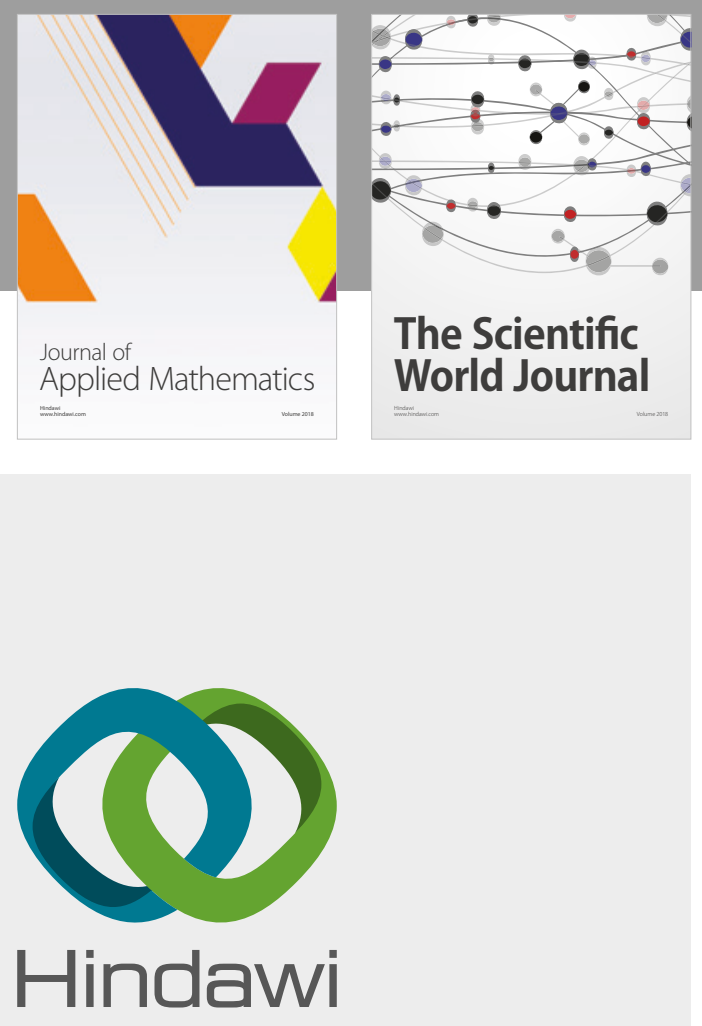

Submit your manuscripts at

www.hindawi.com

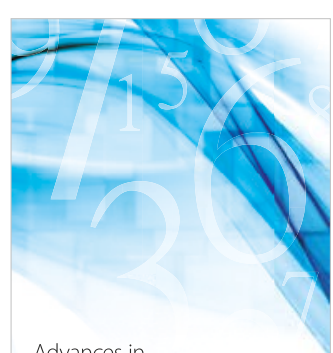

Advances in
Numerical Analysis
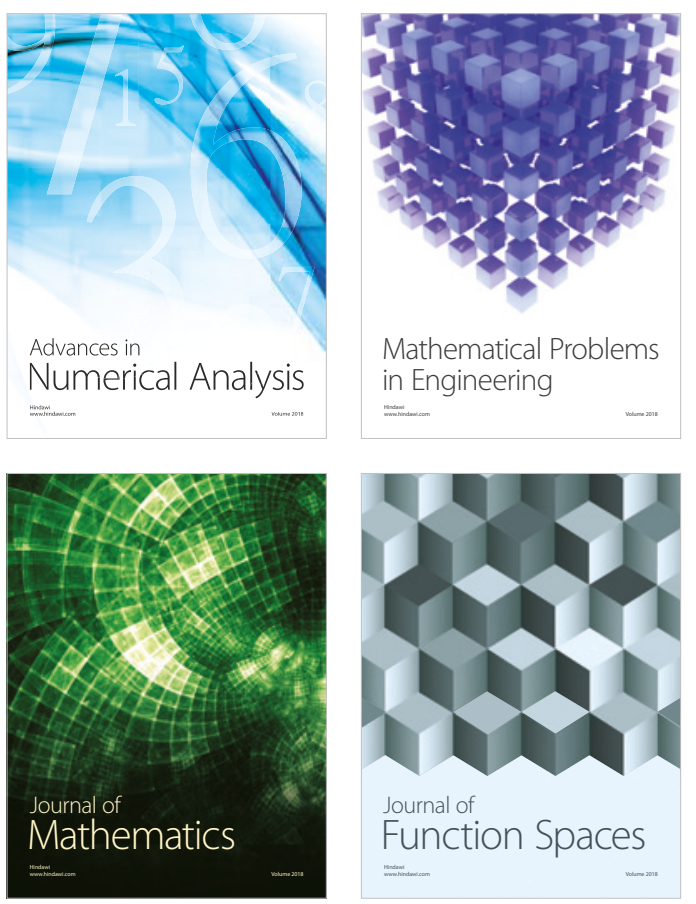

Mathematical Problems in Engineering

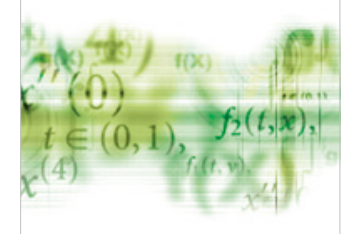

International Journal of

Differential Equations

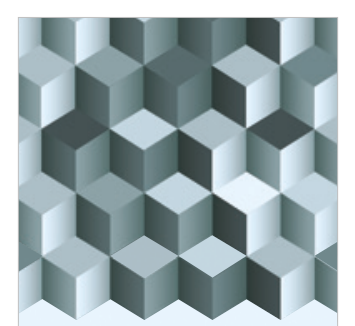

Journal of

Function Spaces

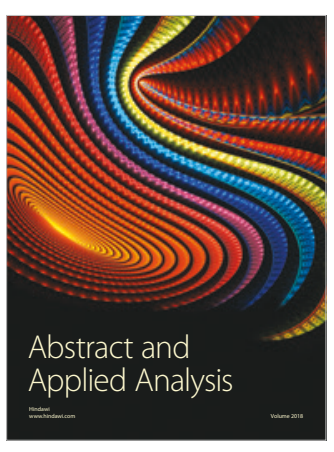

The Scientific

World Journal

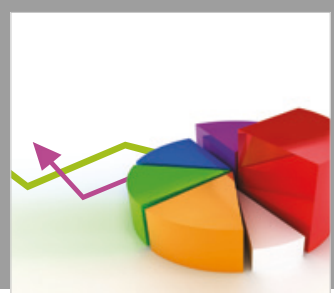

Journal of

Probability and Statistics
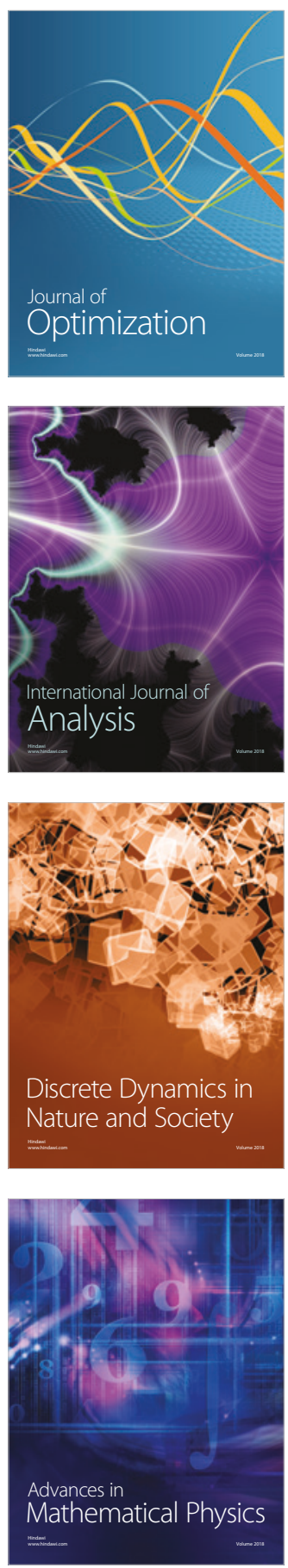